\title{
Atg5 siRNA inhibits autophagy and enhances norcantharidin-induced apoptosis in hepatocellular carcinoma
}

\author{
XUANXUAN XIONG ${ }^{2 *}$, MINGBO WU $^{2 *}$, HAIYAN ZHANG ${ }^{2 *}, \mathrm{JIN} \mathrm{LI}^{1}$, BO LU ${ }^{2}$, YONGGAO GUO $^{2}$, \\ TIAN ZHOU ${ }^{2}$, HAO GUO $^{1}$, RUI PENG $^{3}$, XIANGCHENG LI ${ }^{3}$, QINGZHONG TIAN ${ }^{1}$ and YUN WANG ${ }^{1}$ \\ ${ }^{1}$ Department of Oncological Surgery 2, Xuzhou City Central Hospital, The Affiliated Hospital of the Southeast \\ University Medical School (Xuzhou), The Tumor Research Institute of the Southeast University (Xuzhou), \\ Xuzhou, Jiangsu 221009; ${ }^{2}$ Department Of Gastroenterology 2, Xuzhou City Central Hospital, The Affiliated \\ Hospital of the Southeast University Medical School (Xuzhou), Xuzhou, Jiangsu 221009; ${ }^{3}$ Key Laboratory of \\ Living Donor Liver Transplantation, Ministry of Public Health, Department of Liver Transplantation Center, \\ The First Affiliated Hospital of Nanjing Medical University, Nanjing, Jiangsu 210029, P.R. China
}

Received May 3, 2015; Accepted June 22, 2015

DOI: 10.3892/ijo.2015.3103

\begin{abstract}
Cantharidin is a terpenoid isolated from Chinese blister beetles, and norcantharidin (NCTD) is a demethylated analog of cantharidin. It has been reported that cantharidin and norcantharidin have anticancer activities. Growing evidence suggests that inhibiting autophagy can induce apoptosis in the human hepatoma cell line HepG2. The objective of the present study was to determine whether inhibition of autophagy enhances NCTD-induced apoptosis in HepG2 cells. HepG2 cells were cultured in DMEM containing NCTD. Autophagy was upregulated in the presence of HBSS media supplemented with $\mathrm{Ca}^{2+}$ and $\mathrm{Mg}^{2+}$ and $10 \mathrm{mM}$ HEPES and downregulated in the presence of 3-methyladenine (3-MA) and Atg5 siRNA. Autophagy, cell viability, and the expression of apoptotic proteins were assessed in HepG2 cells. Our data showed that cell apoptosis generally increased after norcantharidin treatment in HepG2 cells. Expression of LC3-II, an autophagosome
\end{abstract}

Correspondence to: Dr Yun Wang or Dr Qingzhong Tian, Department of Oncological Surgery 2, Xuzhou City Central Hospital, The Affiliated Hospital of the Southeast University Medical School (Xuzhou), The Tumor Research Institute of the Southeast University (Xuzhou), 199 Jiefang South Road, Xuzhou, Jiangsu 221009, P.R. China E-mail: wymvp52xx@gmail.com

E-mail: tqz190109@gmail.com

*Contributed equally

Abbreviations: HCC, hepatocellular carcinoma; LC3-II, light chain 3-II; NCTD, norcantharidin; ROS, reactive oxygen species

Key words: autophagy, norcantharidin, hepatocellular carcinoma, apoptosis marker, increased when cells were treated with HBSS media. It also increased cell viability. However, in the presence of 3-MA and Atg5 siRNA, autophagy was inhibited, LC3-II expression decreased and cell apoptosis increased. There was increased expression of Bax, cytochrome $c$, cleaved caspase-3, caspase- 9 and PARP and the mitochondrial membrane potential was disrupted. Additionally, increased apoptosis was accompanied by increased reactive oxygen species (ROS) production. NCTD has anticancer activity, and Atg5 siRNA-mediated downregulation of autophagy enhanced its anticancer actions due to ROS generation and activation of the mitochondrial apoptosis pathway.

\section{Introduction}

Liver cancer is the sixth most frequent cancer and the second leading cause of cancer-related deaths worldwide. Hepatocellular carcinoma (HCC) represents $90 \%$ of all primary liver cancers (1-3). The 5-year survival rate for HCC is $<30 \%$ and the recurrence rate is $\sim 70 \%(4,5)$. Current HCC treatments, including surgical resection, liver transplantation, chemotherapy, or immuno-biological cancer therapies, are typically not very effective.

Cantharidin is a terpenoid isolated from Chinese blister beetles, and norcantharidin (NCTD) is a demethylated analog of cantharidin that has anticancer activity in breast cancer, lung cancer, leukemia, colon and liver cancer. One possible explanation for the anticancer actions of NCTD is its inhibition of protein phosphatases, through which $\mathrm{G} 0 / \mathrm{G} 1$ or $\mathrm{G} 2 / \mathrm{M}$ arrest is triggered. Thus, apoptosis is subsequently induced via ROS generation and the mitochondrial pathway $(6,7)$.

Autophagy, an important pathophysiological process, is crucial for cell development, differentiation, survival and homeostasis. Additionally, autophagy has an important role in liver cancer. Previous studies have shown that autophagy is induced in liver cancer. Inhibition of autophagy was able to 
inhibit tumor cell growth and enhanced HCC cell apoptosis, whereas increased autophagy induced resistance to apoptosis in HCC cells $(8,9)$. NCTD and decreased autophagy could have an anticancer role in liver cancer; however, the mechanisms of autophagy inhibition in liver cancer, as well as the link between NCTD and autophagy, are not well elucidated. Whether NCTD and autophagy coordinate an increase in apoptosis in liver cancer, and elucidation of the downstream apoptotic pathways are not well defined. Because decreased autophagy may induce liver cancer cell apoptosis, NCTD may have an important anticancer role in the liver.

Therefore, the objective of the present study was to examine the relationship between NCTD and autophagy in anticancer activities in the liver. Based on previous data, we hypothesized that autophagy inhibition may enhance NCTD-induced apoptosis in HCC.

\section{Materials and methods}

Cell lines and treatment. The human hepatoma cell line HepG2 was obtained from the Key Laboratory of Living Donor Liver Transplantation, the First Affiliated Hospital of Nanjing Medical University (Nanjing, China). Cells were cultured in Dulbecco's modified Eagle's medium (DMEM) plus 10\% fetal bovine serum (FBS), $100 \mathrm{U} / \mathrm{ml}$ penicillin, and $100 \mathrm{U} / \mathrm{ml}$ streptomycin at $37^{\circ} \mathrm{C}$ with $5 \% \mathrm{CO}_{2}$ (Life Technologies Carlsbad, CA, USA).

Cells were divided into eight groups: i) Sham group: cells were cultured in DMEM without any additional treatment; ii) NCTD group: cells were cultured in DMEM containing NCTD $(2.5,5,10,20$ or $40 \mu \mathrm{g} / \mathrm{ml}$; Sigma-Chemical, St. Louis, MO, USA) for $24 \mathrm{~h}$ (10); iii) $\mathrm{S}$ group: cells were cultured in HBSS media with $\mathrm{Ca}^{2+}$ and $\mathrm{Mg}^{2+}$ supplemented with $10 \mathrm{mM}$ HEPES ( $1 \mathrm{ml} /$ well; Sigma-Aldrich) for $0.5 \mathrm{~h}$ to induce autophagy (11), washed twice with PBS, and cultured for $24 \mathrm{~h}$ in DMEM. iv) SN group: cells were cultured in HBSS media with $\mathrm{Ca}^{2+}$ and $\mathrm{Mg}^{2+}$ supplemented with $10 \mathrm{mM}$ HEPES (1 ml/well) for $0.5 \mathrm{~h}$ to induce autophagy, and washed with PBS before culture for $24 \mathrm{~h}$ in DMEM containing NCTD $(10 \mu \mathrm{g} / \mathrm{ml})$; v) MA group: cells were cultured in DMEM containing 3-MA (10 mmol/l; Sigma-Aldrich) (12); vi) MAN group: cells were cultured in DMEM containing 3-MA (10 mmol/l), washed with PBS before culture for $24 \mathrm{~h}$ in DMEM containing NCTD $(10 \mu \mathrm{g} / \mathrm{ml})$; vii) SS group: cells were transfected with scrambled siRNA (Ambion, Austin, TX, USA) and cultured in DMEM; viii) SSN group: cells were transfected with scrambled siRNA, cultured in DMEM, washed twice with PBS and cultured for $24 \mathrm{~h}$ in DMEM containing NCTD $(10 \mu \mathrm{g} / \mathrm{ml})$.

Small-interfering RNA (siRNA) transfection. Small-interfering RNA(siRNA) against Atg5 and a non-specific scrambled siRNA were purchased from Ambion. All siRNAs were synthesized by Qiagen (Chatsworth, CA, USA). HepG2 cells were cultured in 6-well plates. Lipofectamine 2000 (Invitrogen, Rockville, MD, USA) was mixed into serum-free DMEM containing siRNA1, siRNA2 or scrambled RNA. Additionally, mock controls were transfected with Lipofectamine 2000 alone, incubated at room temperature for $20 \mathrm{~min}$ and distributed into duplicate wells. Transfections were performed at $37^{\circ} \mathrm{C}$ in $5 \% \mathrm{CO}_{2}$. Media were replaced with DMEM containing $10 \%$ heat-inactivated FBS for 4-6 h. After $2 \mathrm{~h}$, cells were treated as indicated.

Reverse transcription-quantitative real-time polymerase chain reaction $(R T-q P C R)$. We isolated and purified total RNA from cells by using TRIzol reagent (Invitrogen) and synthesized cDNA using the PrimeScript RT Master Mix. cDNA $(2 \mu \mathrm{l})$ was used as a template in a $20-\mu \mathrm{l}$ reaction. Primers were synthesized by Invitrogen (Shanghai, China). The primers for Atg5 were: 5'-TTCTCAAAATATACTGTTTC-3' (sense) and 5'-TATTATGTATCACAAATGG-3' (antisense). $\beta$-actin was used as a control. The primers for $\beta$-actin were: 5'-TCACCCACACTGTGCCCATCTACGA-3' (sense) and 5'-CAGCGGAACCGCTCATTGCCAATGG-3' (antisense). For RT-PCR, we used the following cycles: $95^{\circ} \mathrm{C}$ for $30 \mathrm{sec}$, 40 cycles of $95^{\circ} \mathrm{C}$ for $5 \mathrm{sec}$ and $60^{\circ} \mathrm{C}$ for $31 \mathrm{sec}$. The dissociation stage was $95^{\circ} \mathrm{C}$ for $15 \mathrm{sec}, 60^{\circ} \mathrm{C}$ for $1 \mathrm{~min}$ and $95^{\circ} \mathrm{C}$ for $15 \mathrm{sec}$.

Western blot analyses. HepG2 cells were washed with cold PBS and homogenized in RIPA lysis buffer in the presence of $1 \%$ (v/w) protease inhibitor (Pierce Biotechnology, Rockford, IL, USA). Cell lysates were then shaken at $4^{\circ} \mathrm{C}$ for $1 \mathrm{~h}$ and centrifuged for $1 \mathrm{~h}$ at $40,000 \mathrm{x} \mathrm{g}$ at $4^{\circ} \mathrm{C}$. For cytochrome $c$ analyses, cell pellets were cultured in HEPES buffer containing $250 \mathrm{mM}$ sucrose and homogenized. Homogenates were centrifuged for $15 \mathrm{~min}$ at $800 \mathrm{x} \mathrm{g}$ at $4^{\circ} \mathrm{C}$ and supernatants were centrifuged for $15 \mathrm{~min}$ at $10,000 \mathrm{x} \mathrm{g}$ at $4^{\circ} \mathrm{C}$. Finally, the mitochondrial pellets and cytosolic fractions were collected (13). Protein concentrations were determined using BCA protein assays. Protein lysates $(50 \mu \mathrm{g})$ were separated by $12 \%(\mathrm{w} / \mathrm{v})$ SDS-PAGE, and proteins were transferred to polyvinylidene difluoride (PVDF) membranes (Millipore, Bedford, MA, USA). Membranes were incubated with the primary antibodies (caspase antibodies were obtained from Abcam, Cambridge, UK; the LC3-II antibody was from Novus Biologicals, Littleton, CO, USA) overnight at $4^{\circ} \mathrm{C}$, incubated for $1 \mathrm{~h}$ with the secondary antibody (1:4,000, $\beta$-actin; Abcam), and visualized using an enhanced chemiluminesence (ECL) detection kit (Pierce Biotechnology). For statistical analyses, the gray value of each protein was measured using Image-Pro Plus 6.0.

Immunofluorescence. HepG2 cells on coverslips were fixed in $4 \%$ paraformaldehyde for $20 \mathrm{~min}$. Cells were then blocked in $10 \%$ BSA for $1 \mathrm{~h}$, followed by overnight incubation with the primary antibody (LC3II in 10\% BSA). Cells were incubated with the secondary antibody (1:100) for $1 \mathrm{~h}$ at $37^{\circ} \mathrm{C}$ and then mounted using 4',6-diamidino-2-phenylindole (DAPI) mounting media. We evaluated the cells for autophagy by randomly choosing ten visual fields and measuring the optical density. Semi-quantitative analyses were performed using Image-Pro Plus 6.0.

Annexin V/propidium iodide (PI) staining assays. We quantified apoptosis in HepG2 cells using the Annexin V-FITC kit. Cells were washed twice with ice-cold PBS and resuspended in binding buffer $(400 \mu \mathrm{l})$ at a concentration of $5 \times 10^{5}$ cells $/ \mathrm{ml}$. After incubation, $5 \mu \mathrm{l}$ Annexin V-FITC and $5 \mu \mathrm{l}$ PI were added. Tubes were gently mixed on a vortex and incubated 

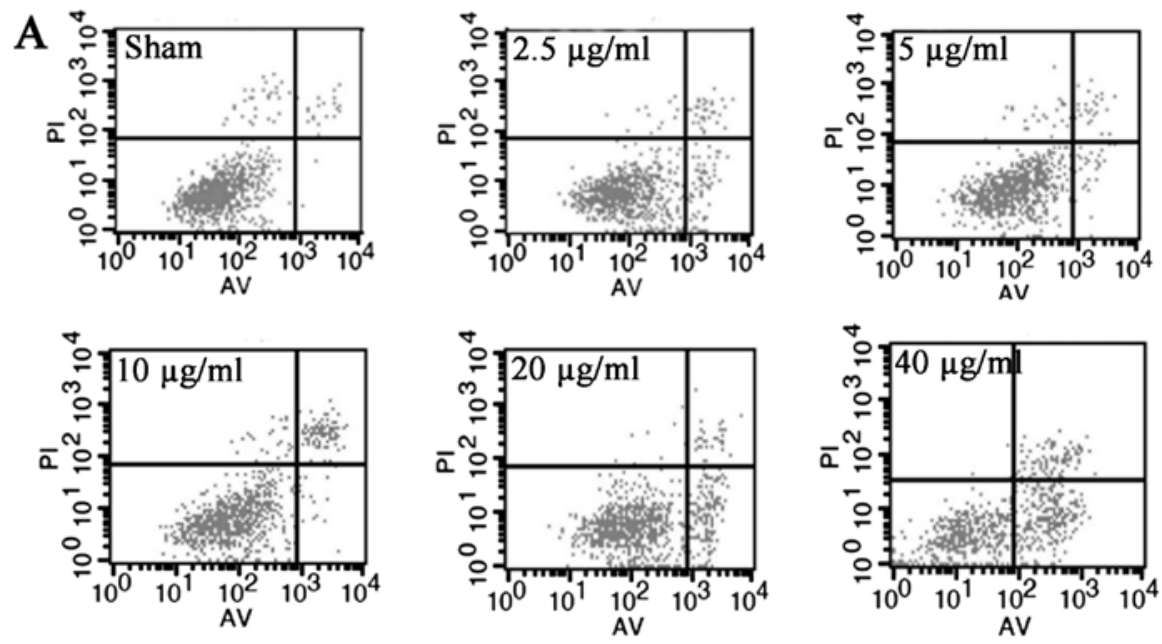

B

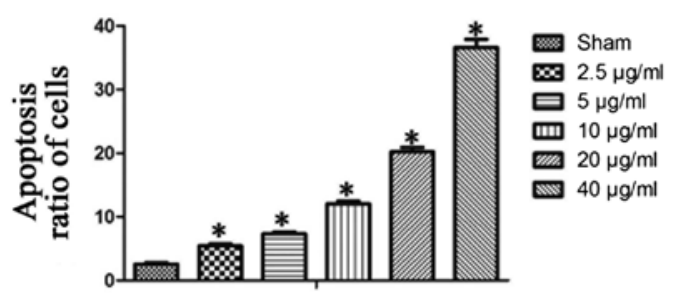

Figure 1. NCTD induces apoptosis in HepG2 cells. (A and B) NCTD pre-treatment of HepG2 cells increased apoptosis, and NCTD dose-dependently increased the apoptotic ratios of the cells. The basal apoptotic population of the sham group was $2.6 \pm 0.5 \%$. After treatment with $2.5,5,10,20$ and $40 \mu \mathrm{g} / \mathrm{ml} \mathrm{NCTD} \mathrm{for}$ $24 \mathrm{~h}$, the apoptotic rates increased to $5.5 \pm 0.7,7.4 \pm 0.6,12.1 \pm 0.8,20.2 \pm 1.5$ and $36.6 \pm 2.8 \%$, respectively $\left({ }^{*} \mathrm{P}<0.05\right.$ compared with the sham group).

for $15 \mathrm{~min}$ in the dark at $4^{\circ} \mathrm{C}$. Samples were analyzed using a FACSCalibur flow cytometer (BD Biosciences, San Jose, CA, USA) and CellQuest software (BD Biosciences).

ROS production analyses. Intracellular ROS levels were detected by flow cytometry using 2',7'-dichlorodihydrofluorescein diacetate (DCFH-DA; Nanjing KeyGen Biotech Co., Ltd., Nanjing, China). HepG2 cells were washed three times with PBS solution, and cultured in serum-free DMEM containing DCFH-DA $(100 \mu \mathrm{M})$ for $30 \mathrm{~min}$ in the dark at $37^{\circ} \mathrm{C}$. Cells were then washed in serum-free DMEM and treated with pancreatic enzymes. DCF fluorescence was detected at an excitation wavelength of $488 \mathrm{~nm}$ and an emission wavelength of $525 \mathrm{~nm}$.

Mitochondrial membrane potential ( $\Delta \Phi m)$ assays. The loss of mitochondrial membrane potential was monitored using the membrane permeable dye JC-1 (Beyotime Institute of Biotechnology, Haimen, China). At high $\Delta \Phi m$, JC-1 shows red fluorescence, and the ratio between green and red fluorescence provides an estimate of $\Delta \Phi \mathrm{m}$. Briefly, JC-1 dyeing liquid was added to each well $(1 \mathrm{ml} /$ well $)$ and cells were incubated at $37^{\circ} \mathrm{C}$ for $20 \mathrm{~min}$. Cells were then washed twice with dyeing buffer (1X) and cultured in DMEM. Finally, cells were observed using fluorescence microscopy.

Statistical analyses. Data are expressed as the mean \pm standard deviation. Differences between groups were evaluated using variance, q-tests and Student's t-tests. SPSS 11.0 software was used for all statistical analyses, and $\mathrm{P}<0.05$ was considered statistically significant.

\section{Results}

NCTD induces apoptosis in HepG2 cells. First, we examined apoptosis in HepG2 cells. We found that NCTD dose-dependently increased the apoptosis ratios in HepG2 cells. The basal apoptotic population of the sham group was $2.6 \pm 0.5 \%$. After treatment for $24 \mathrm{~h}$ with $2.5,5,10,20$ and $40 \mu \mathrm{g} / \mathrm{ml} \mathrm{NCTD,}$ the apoptotic rates increased to $5.5 \pm 0.7,7.4 \pm 0.6,12.1 \pm 0.8$, $20.2 \pm 1.5$ and $36.6 \pm 2.8 \%$, respectively (Fig. 1). Based on these data, $10 \mu \mathrm{g} / \mathrm{ml}$ NCTD was used in all subsequent experiments.

Autophagy counteracts NCTD-induced apoptosis. Next, we examined LC3-II expression and found that HBSS pretreatment increased LC3-II protein expression. Decreased apoptotic rates were observed in the HBSS and NCTD (SN) group compared with the sham group. HBSS-treated HepG2 cells showed increased LC3-II protein expression in western blotting and immunostaining analyses (Figs. 2B and 3; $\mathrm{P}<0.05)$. Moreover, Annexin V/PI staining indicated that the induction of autophagy in the HBSS and NCTD (SN) group decreased the rates of early and late apoptosis in HepG2 cells compared with the sham group (Fig. $2 \mathrm{C} ; \mathrm{P}<0.05$ ).

Inhibition of autophagy induces HepG2 cell apoptosis. 3-MA and Atg5 siRNA were used in HepG2 cells to inhibit LC3-II expression. We observed decreased Atg5 gene expression in the Atg5 siRNA and NCTD (SSN) group compared with the sham group (Fig. 2A; P<0.05). In addition, LC3-II expression was inhibited in the 3-MA and NCTD (MAN) or Atg5 siRNA and NCTD (SSN) groups compared with the sham group as shown by western blotting and immunostaining assays 


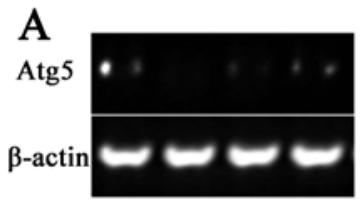

Sham S1 S2 SS

C

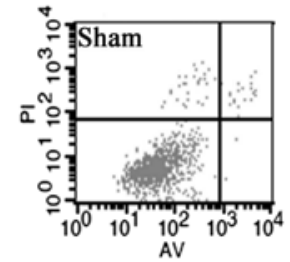

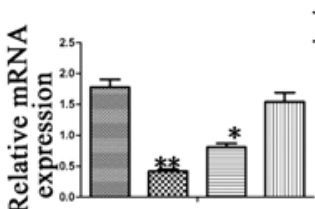

Sham S1 S2 SS
B
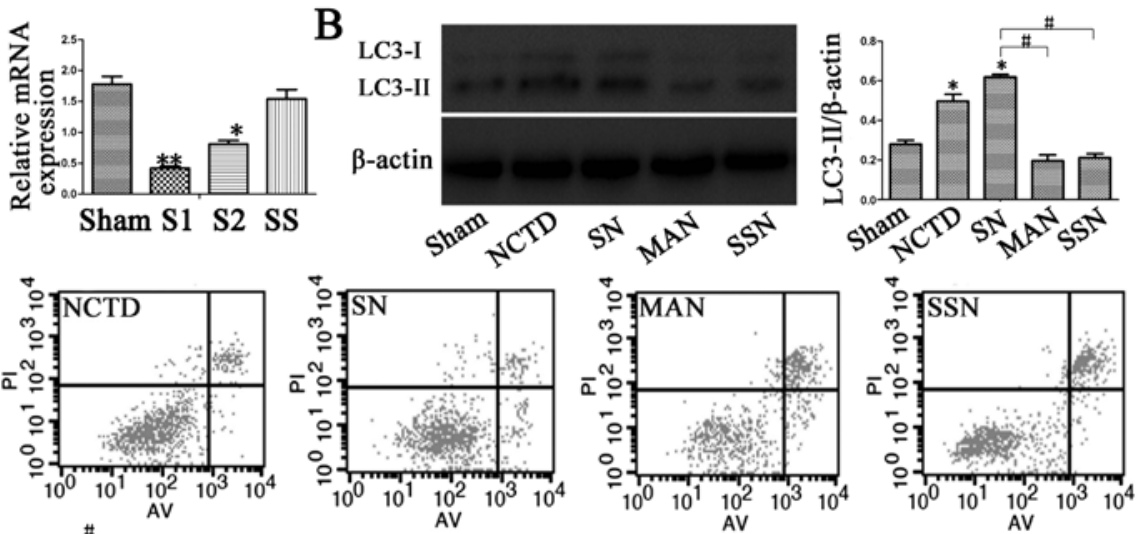

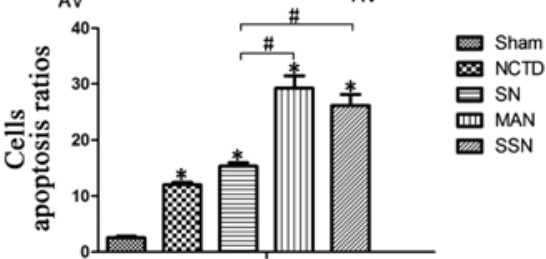

Figure 2. Inhibition of autophagy induces HepG2 cell apoptosis. (A) Atg5 gene expression decreased in the SSN group compared with the sham group based on RT-qPCR analyses. (B) HBSS pre-treatment increased LC3-II protein expression, whereas LC3-II expression was inhibited when 3-MA and Atg5 siRNA were added to HepG2 Cells. (C and D) Annexin V/PI staining showed that the induction of autophagy in the SN group decreased the rates of early and late apoptosis in HepG 2 cells compared with the sham group. The inhibition of autophagy increased the apoptosis rates of HepG2 cells $\left({ }^{*} \mathrm{P}<0.05\right.$ and ${ }^{* *} \mathrm{P}<0.01$ compared with the sham group; ${ }^{\#} \mathrm{P}<0.05$ compared with the $\mathrm{SN}$ group).
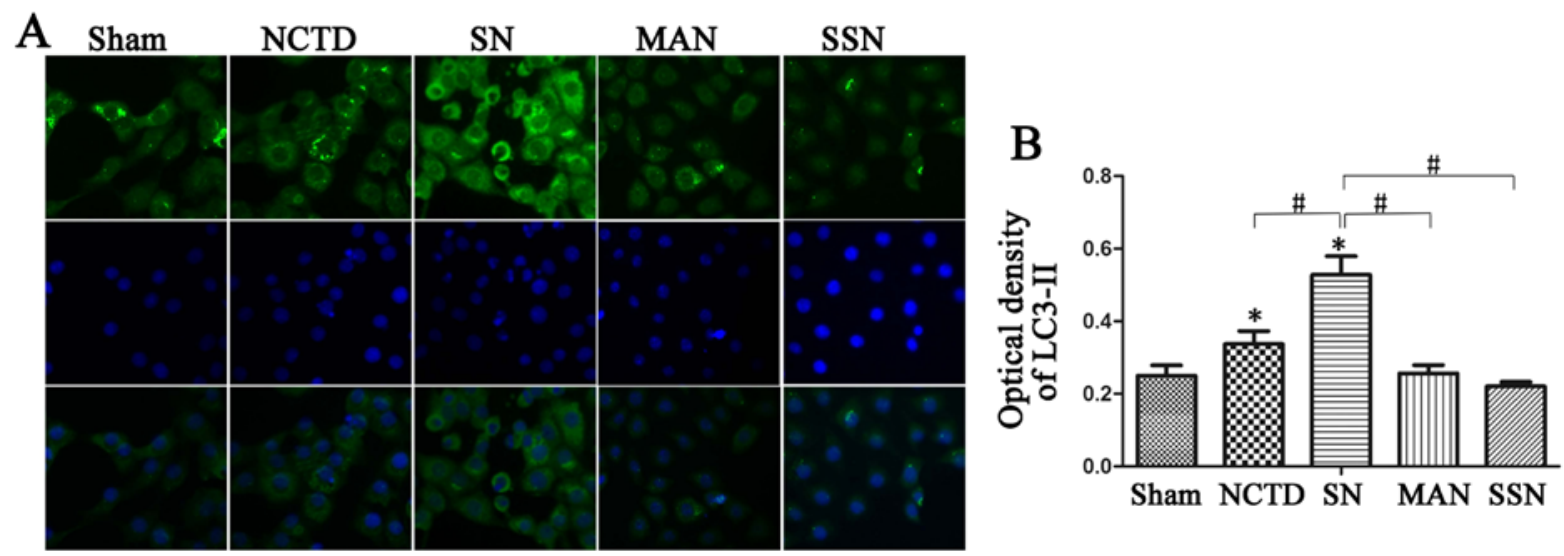

Figure 3. LC3-II protein expression is decreased in cells treated with NCTD. (A and B) Decreased LC3-II staining in the MAN and SSN groups by immunofluorescence. The photometric values also showed decreased LC3-II staining in the MAN and SSN groups; however, it was increased in the SN group ( $\mathrm{P}<0.05$ compared with the sham group; ${ }^{\text {}} \mathrm{P}<0.05$ compared with the $\mathrm{SN}$ group).

(Figs. 2B and 3; $\mathrm{P}<0.05)$. Finally, we evaluated the apoptotic ratios and found that autophagy inhibition increased the rate of apoptosis in HepG2 cells (Fig. 2C; $\mathrm{P}<0.05$ ).

NCTD induces ROS generation and autophagy inhibition enhances this activity. To further examine the role of NCTD and autophagy, we evaluated ROS levels in cells after the various treatments. We found increased intracellular ROS generation in cells treated with NCTD. Moreover, autophagy inhibition further enhanced ROS generation compared with the NCTD group. However, when autophagy was induced, ROS levels were downregulated (Fig. 4).

Autophagy inhibition increases Bax, cytochrome $c$, caspase-3, caspase-9 and PARP protein expression and decreases Bcl-2 expression. To further elucidate the mechanisms of apoptosis, we examined the expression of apoptotic proteins. As shown in Fig. 5, downregulation of autophagy increased Bax and cytosolic cytochrome $c$ protein expression compared with the sham group. However, the increase was abrogated when autophagy was induced in the HBSS and NCTD (SN) group $(\mathrm{P}<0.05)$. Additionally, mitochondrial cytochrome $c$ and $\mathrm{Bcl}-2$ protein expression was downregulated in the HBSS and NCTD (SN) group compared with the sham, 3-MA and NCTD (MAN) or Atg5 siRNA and NCTD $(\mathrm{SSN})$ groups $(\mathrm{P}<0.05)$.

Next, we evaluated the cleavage of the caspase-3, caspase- 8 and caspase-9. Expression of cleaved caspase-3 and caspase-9 was rapidly increased in the 3-MA and NCTD (MAN) or Atg5 siRNA and NCTD (SSN) groups compared with the sham 

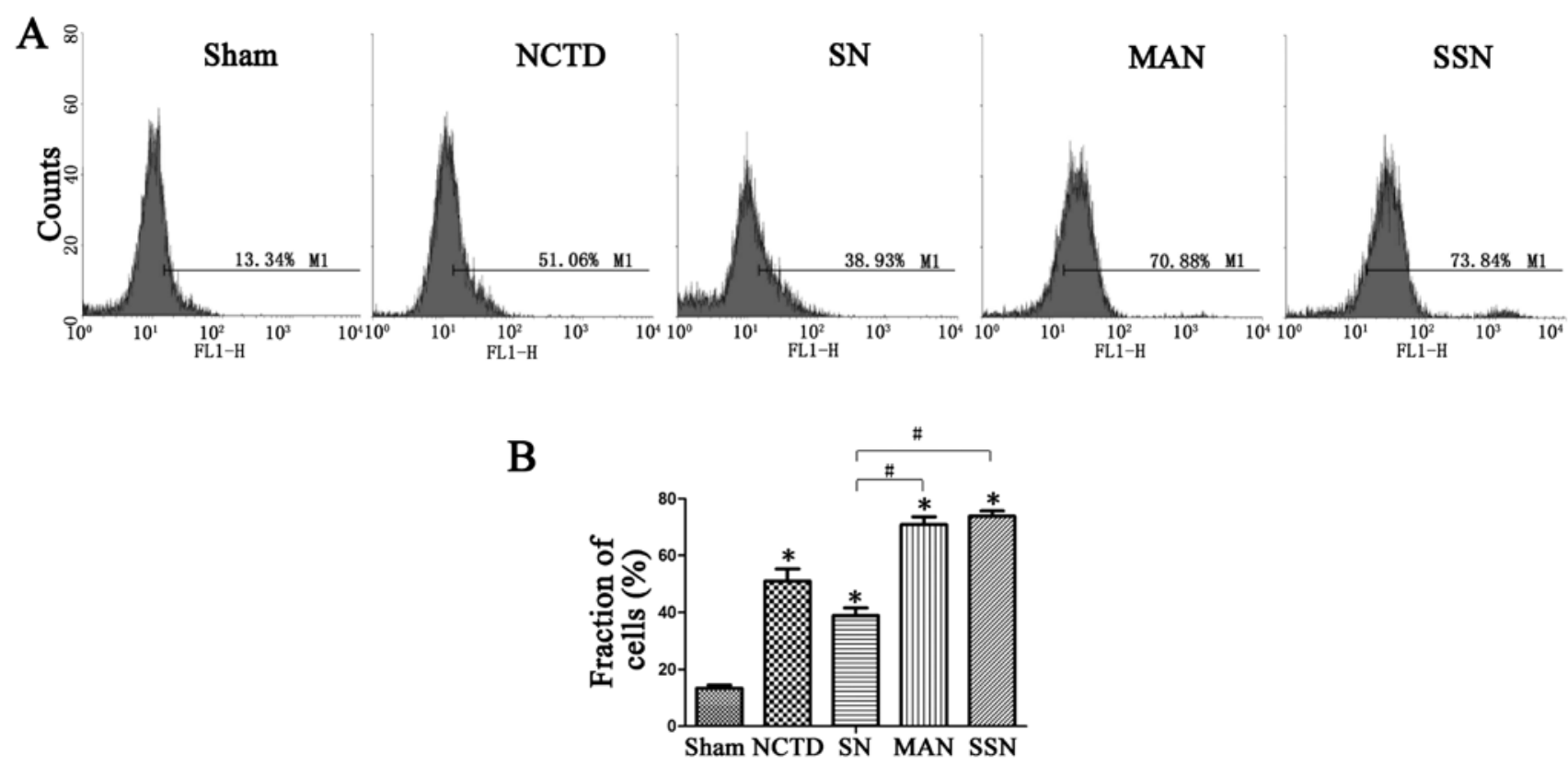

Figure 4. NCTD induced ROS generation and autophagy inhibition enhanced this activity. (A and B) There is increased intracellular ROS generation in NCTD-treated cells. Inhibition of autophagy further enhanced ROS generation compared with the NCTD group. However, when autophagy was induced, ROS levels were downregulated ( $\mathrm{P}<0.05$ compared with the sham group; ${ }^{*} \mathrm{P}<0.05$ compared with the $\mathrm{SN}$ group).
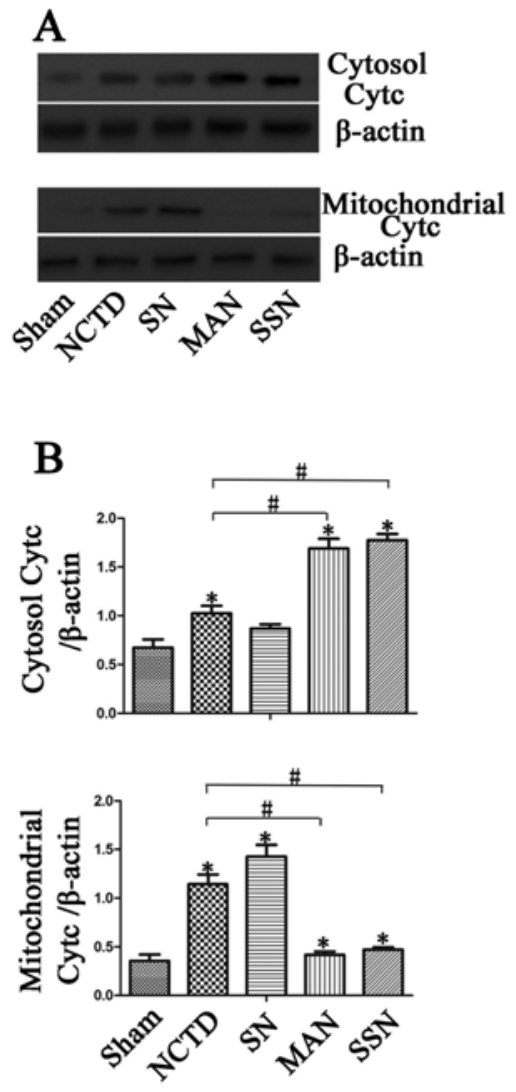
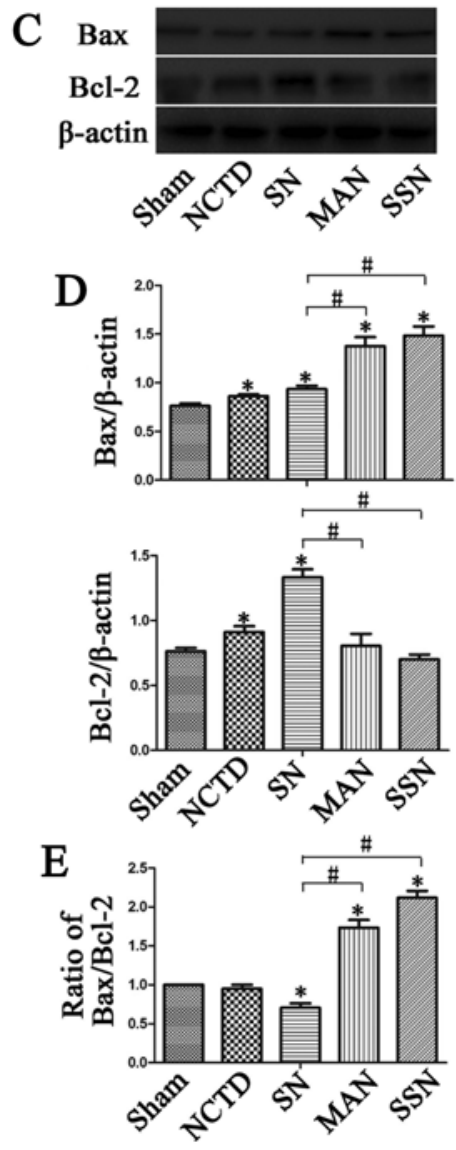

Figure 5. Autophagy inhibition induced Bax, cytochrome $c$, caspase-3, caspase-9 and PARP protein expression, and reduced Bcl-2 expression. (A and B) After treatment with NCTD, cytosolic cytochrome $c$ protein expression increased compared with the sham group. However, when autophagy was downregulated, cytochrome $c$ protein expression was significantly increased in the cytosolic fraction, but the increase was abrogated when autophagy was induced in the SN group ( $\left(\mathrm{P}<0.05\right.$ compared with the sham group; ${ }^{*} \mathrm{P}<0.05$ compared with the $\mathrm{SN}$ group). (C and $\left.\mathrm{D}\right) \mathrm{Bax}$ protein expression was upregulated in the MAN and SSN groups compared with the sham and SN group. The highest Bcl-2 protein expression was observed in the SN group ("P<0.05 compared with the sham group; ${ }^{~} \mathrm{P}<0.05$ compared with the $\mathrm{SN}$ group). (E) The ratio of Bax/Bcl-2 was highest in the MAN and SSN groups (" $\mathrm{P}<0.05$ compared with the control sham; ${ }^{\#} \mathrm{P}<0.05$ compared with the $\mathrm{SN}$ group). 

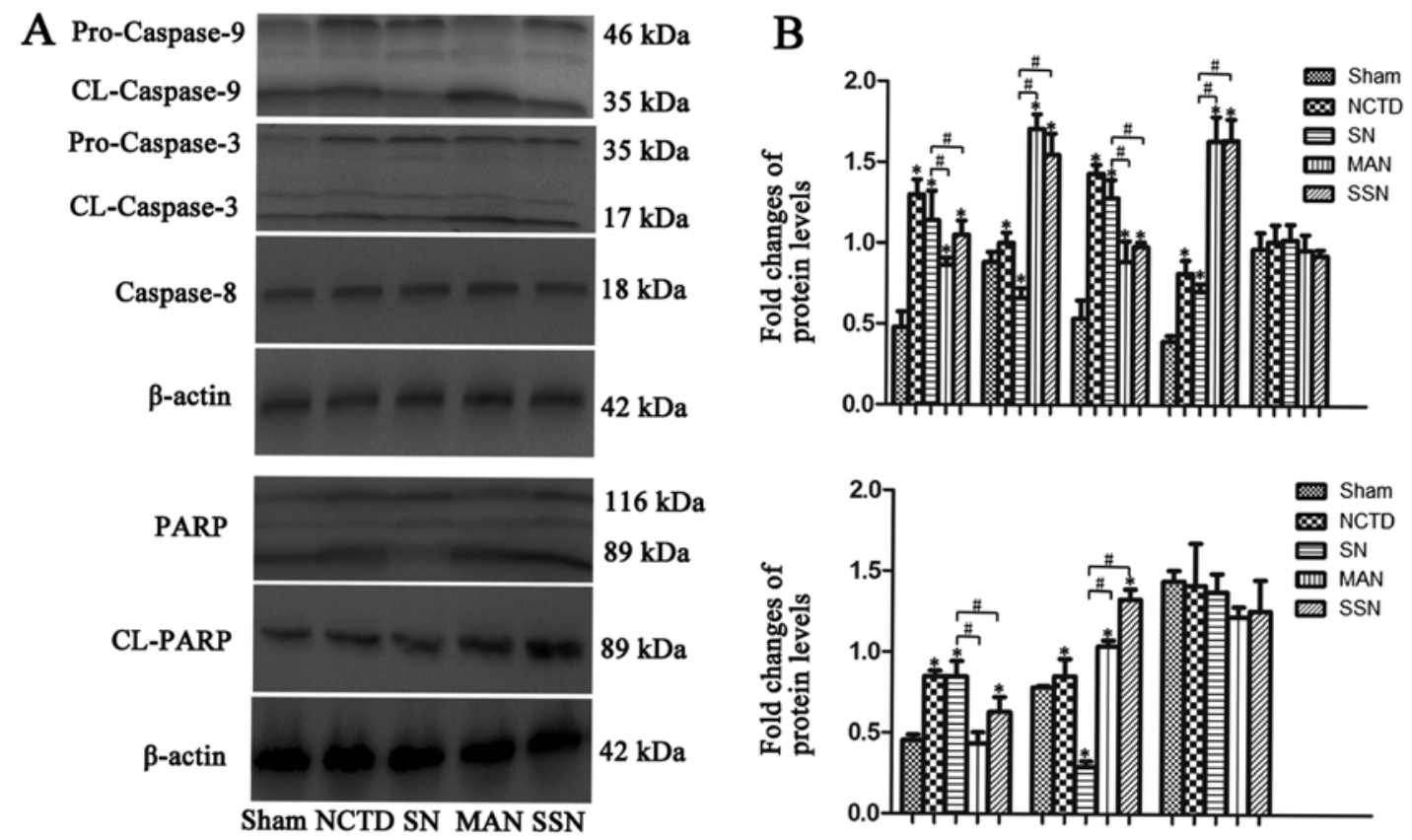

Figure 6. Inhibition of autophagy in HepG2 cells induced cleavage of caspase-3, caspase-9 and PARP. (A) The expression of cleaved caspase-3 and caspase-9 in the cytosolic fraction increased rapidly after NCTD treatment. Compared to cells in the NCTD group, the cleavage of caspase- 3 and caspase- 9 increased when autophagy was inhibited $(\mathrm{P}<0.05)$. There were no significant differences in caspase- 8 expression in all groups. Furthermore, induction of autophagy attenuated the increased cleavage of caspase-3 and caspase-9. Inhibition of autophagy in HepG2 cells promoted PARP cleavage ("P $<0.05$ compared with the sham group; ${ }^{\text {"}} \mathrm{P}<0.05$ compared with the $\mathrm{SN}$ group). (B) Fold-schanges of protein levels; each group of bars indicates a different protein. The order of the proteins shown is according to the order shown in (A).

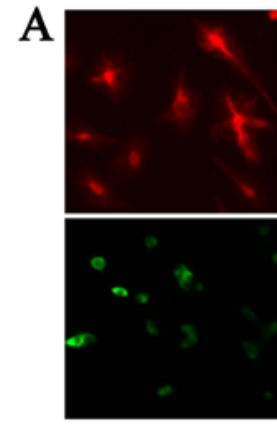

Sham

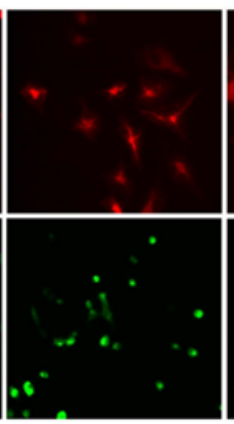

NCTD

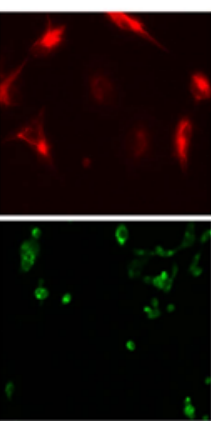

SN

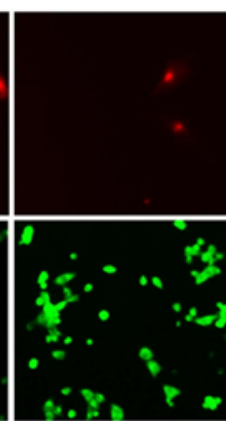

MAN

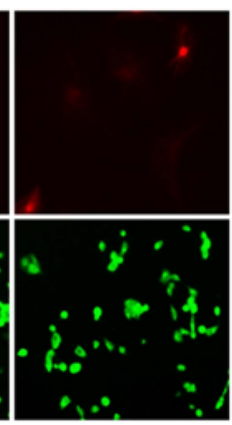

SSN

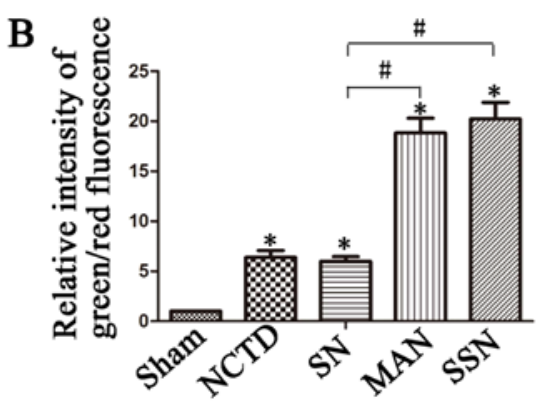

Figure 7. Mitochondrial membrane potential disturbances were observed in the MAN and SSN groups. (A and B) The increase in green fluorescent cells indicated increased mitochondrial membrane potential disturbance. There was more red fluorescence in the sham and SN groups and more green fluorescence in the MAN and SSN groups ( $\mathrm{P}<0.05$ compared with the sham group; ${ }^{*} \mathrm{P}<0.05$ compared with the $\mathrm{SN}$ group).

group; however, the increase was abrogated in the HBSS and NCTD (SN) group. We observed no significant changes in the cleaved caspase- 8 expression in any of the groups (Fig. 6; $\mathrm{P}<0.05)$. Inhibition of autophagy in HepG2 cells also promoted PARP cleavage (Fig. 6; $\mathrm{P}<0.05$ ).
The mitochondrial membrane potential is disturbed in cells treated with NCTD. Increased green fluorescence in cells indicates increased mitochondrial membrane potential disturbance. In the present study, we observed more red fluorescence in the sham and HBSS and NCTD (SN) cells, with more green 
fluorescence in the 3-MA and NCTD (MAN) or Atg5 siRNA and NCTD (SSN) groups (Fig. 7; $\mathrm{P}<0.05$ ). These data indicate that there is increased mitochondrial membrane potential disturbance in cells treated with NCTD, and inhibition of autophagy enhanced this effect.

\section{Discussion}

In the present study, we found that NCTD induced HepG2 cell apoptosis. Decreased apoptotic ratios were detected when autophagy was induced in these cells. Moreover, inhibition of autophagy in HepG2 cells induced HepG2 cell apoptosis.

To further determine the mechanism of apoptosis, we examined expression of cytochrome $c, \mathrm{Bax}, \mathrm{Bcl}-2$, caspase-3, caspase- 8 and caspase-9 protein. We found that inhibition of LC3-II expression using 3-MA and Atg5 siRNA increased HepG2 cell apoptosis ratios as shown by increased expression of cytosolic cytochrome $c$ and Bax. In addition, there was increased cleavage of caspase-3 and -9. However, when autophagy was induced, less caspase- 3 and -9 was cleaved, and mitochondrial cytochrome $c$ protein and Bcl-2 protein expression increased. Finally, enhanced ROS generation was observed in cells where autophagy was downregulated. When autophagy was induced, ROS levels were downregulated. Based on our current data and data from previous reports, we hypothesize that decreasing autophagy increases HepG2 cell apoptosis partly through ROS generation and activation of the mitochondrial apoptotic pathway.

Hepatocellular carcinoma (HCC) is the most common type of liver cancer and is one of the leading causes of cancer deaths worldwide. Because of the complex mechanisms of HCC, effective therapies toward HCC have not significantly improved $(1,2)$. As a heterogeneous tumor, HCC involves multiple pathways and molecular alterations (14). With medical technology advances, there are increasing number of new treatments towards HCC, including surgical resection, liver transplantation and chemotherapy or immuno-biological cancer therapy. Cantharidin, which is extracted from the Chinese blister beetle, has been used for many years in Chinese medicine to treat tumors. However, because of nephrotoxic and other side effects, norcantharidin (NCTD) was synthesized in an effort to reduce toxicity. Currently, NCTD is widely used in the treatment of tumors $(7,15)$.

It has been shown that NCTD has antitumor roles in various experimental animal models and extends the life of some of these animals. The mechanism of action of NCTD involves inhibition of protein and nucleic acid synthesis. $\mathrm{N}$-hydroxycantharidimide, which is synthesized by cantharidin, has certain curative effects in liver cancer (16). Previous data showed that NCTD could induce cytotoxicity in HepG2 cells by inducing apoptosis (10); therefore, in the present study, we evaluated HepG2 cell apoptosis. The basal apoptotic population of the sham group was $0.3 \pm 0.1 \%$. After treatment with $2.5,5,10,20$ and $40 \mu \mathrm{g} / \mathrm{ml} \mathrm{NCTD}$, the apoptotic rate increased to $5.5 \pm 0.7,7.4 \pm 0.6,12.1 \pm 0.8,20.2 \pm 1.5$ and $36.6 \pm 2.8 \%$, respectively. These data indicate that NCTD increases apoptosis in HepG2 cells, and further demonstrate the dose-dependence of NCTD on the apoptotic ratios in these cells.

Our previous studies showed that miR-101 may enhance cisplatin-induced apoptosis in HCC cells, and the possible mechanism involved inhibition of autophagy via targets including RAB5A, STMN1 and ATG4D (17). Other studies have reported that autophagy affects liver cancer. Autophagy is a process whereby cytoplasmic macromolecules or organelles are degraded in lysosomes to maintain the cellular balance. Previous data indicate that autophagy functions as a survival mechanism and elicits protective effects against tissue and cell injury (18). In general, it has been noted that autophagy has dual functions, it can lead to autophagic cell death under certain conditions but also can keep cells alive under stressful, 'life-threatening' conditions. It has been shown that inhibition of autophagy induced apoptosis of tumor cells, and knockdown of TP53-induced glycolysis regulator (TIGAR) expression could enhance the antitumor effects of epirubicin by increasing cellular ROS levels, activating apoptotic pathways, and inhibiting autophagy-enhanced epirubicin-induced apoptosis (19). Like the above chemotherapeutic drug, in the present study, we found that NCTD was able to induce HepG2 cell apoptosis, and that inhibition of autophagy in HepG2 cells induced apoptosis. Taken together, these data suggest that autophagy leads to autophagic cell death in HepG2 cells.

Apoptosis can be initiated via alternative signaling pathways: the intrinsic pathway, which is also called the mitochondria-mediated pathway and the extrinsic or death receptor-mediated pathway. Mitochondria play a critical role in apoptotic processes, including drug-induced apoptosis $(20,21)$. Reports have shown that NCTD could be used to prevent and treat cancer, likely due to increased apoptosis via ROS generation and the mitochondrial pathway (22). Mitophagy is a process that refers to mitochondria degradation via autophagy. Selective degradation of mitochondria is highly regulated by various molecules, and is crucial in cell apoptosis (23).

Therefore, to further dissect the mechanism of NCTD action, we focused on the apoptotic pathways. It was reported that the anti-apoptotic protein $\mathrm{Bcl}-2$ and the pro-apoptotic protein Bax are crucial for initiation of the mitochondrial death cascade. We observed downregulation of Bax and upregulation of $\mathrm{Bcl}-2$ in the autophagy-induced group. However, inhibition of LC3-II expression using 3-MA and Atg5 siRNA may prevent changes in Bax and Bcl-2 expression. Moreover, cytosolic cytochrome $c$ protein expression was induced and mitochondrial cytochrome $c$ protein expression decreased when autophagy was inhibited. Cleaved caspase-3 and caspase- 9 were also detected, indicating that activation of caspase- 3 and caspase- 9 are important in the mitochondrial apoptotic pathway. The present study found increased cleavage of caspase-3 and caspase-9 in the 3-MA and NCTD (MAN) or Atg 5 siRNA and NCTD (SSN) groups in which autophagy was decreased. However, the cleavage of caspase- 3 and caspase- 9 was decreased in the sham and HBSS and NCTD (SN) cells in which autophagy was increased.

We also evaluated PARP protein levels. The PARP protein is an important apoptosis protein, which is always cleaved by activated caspase-3. In the 3-MA and NCTD (MAN) or Atg 5 siRNA and NCTD (SSN) groups, we observed increased PARP protein cleavage from the full-length $116 \mathrm{kDa}$ form to its cleaved $89 \mathrm{kDa}$ form. However, PARP was not cleaved in the SN group. Finally, we examined the mitochondrial membrane potential. We detected more red fluorescence in the control and SN groups, but more green fluorescence in 
the 3-MA and NCTD (MAN) or Atg5 siRNA and NCTD (SSN) groups, indicating disturbed mitochondrial membrane potential. Our data suggest that the inhibition of autophagy increased NCTD-induced apoptosis, which may occur via a mitochondria-mediated pathway.

ROS are a series of reactive oxygen species produced during metabolic processes that are important in tumor occurrence, development and recurrence. Recent studies have shown that ROS can accelerate tumor cell death and have antitumorigenic roles (24). Drugs that increase ROS levels have been produced and used in clinical trials $(24,25)$. Some studies have reported that ROS act as a second messenger in apoptosis. The generation of ROS can induce mitochondrial membrane potential disturbance, thereby contributing to mitochondrial damage and leading to cell death by acting as an apoptotic signaling molecule (26). We found that NCTD induced ROS production in HepG2 cells. The inhibition of autophagy caused an NCTDinduced increase in ROS in HepG2 cells, and the increase in ROS was abolished or attenuated by increasing autophagy.

In conclusion, we demonstrate that NCTD induced HepG2 cell apoptosis. The inhibition of autophagy in these cells also induced apoptosis. Inhibiting LC3-II expression by 3-MA and Atg 5 siRNA also has pro-apoptotic effects via the mitochondrial apoptotic pathway, and enhanced ROS generation was observed in cells where autophagy was downregulated. Therefore, we propose that decreased autophagy increases HepG2 cell apoptosis, partly via ROS generation and activation of the mitochondrial apoptotic pathway. However, the exact relationship between autophagy and the mitochondrial apoptotic pathway, as well as the function of autophagy in apoptosis, require further study.

\section{Acknowledgements}

The present study was supported by grants from the City Subject Foundation of Xuzhou (KC14SH020) and the Xuzhou City Central Hospital (XZS2013018).

\section{References}

1. Dubois-Pot-Schneider H, Fekir K, Coulouarn C, Glaise D, Aninat C, Jarnouen K, Le Guével R, Kubo T, Ishida S, Morel F, et al: Inflammatory cytokines promote the retrodifferentiation of tumor-derived hepatocyte-like cells to progenitor cells. Hepatology 60: 2077-2090, 2014.

2. Su YH, Lin SY, Song W and Jain S: DNA markers in molecular diagnostics for hepatocellular carcinoma. Expert Rev Mol Diagn 14: 803-817, 2014.

3. White JA, Redden DT, Bryant MK, Dorn D, Saddekni S, Aal AKA, Zarzour J, Bolus D, Smith JK, Gray S, et al: Predictors of repeat transarterial chemoembolization in the treatment of hepatocellular carcinoma. HPB (Oxford) 16: 1095-1101, 2014.

4. Ferlay J, Soerjomataram I, Dikshit R, Eser S, Mathers C, Rebelo M, Parkin DM, Forman D and Bray F: Cancer incidence and mortality worldwide: Sources, methods and major patterns in GLOBOCAN 2012. Int J Cancer 136: E359-E386, 2015.

5. Bruix J and Sherman M; American Association for the Study of Liver Diseases: Management of hepatocellular carcinoma: An update. Hepatology 53: 1020-1022, 2011.

6. Yeh $\mathrm{CH}$, Yang YY, Huang YF, Chow KC and Chen MF: Induction of apoptosis in human Hep3B hepatoma cells by norcantharidin through a p53 independent pathway via TRAIL/DR5 signal transduction. Chin J Integr Med 18: 676-682, 2012.
7. Han W, Wang S, Liang R, Wang L, Chen M, Li H and Wang Y: Non-ionic surfactant vesicles simultaneously enhance antitumor activity and reduce the toxicity of cantharidin. Int $\mathrm{J}$ Nanomed 8: 2187-2196, 2013.

8. Rautou PE, Mansouri A, Lebrec D, Durand F, Valla D and Moreau R: Autophagy in liver diseases. J Hepatol 53: 1123-1134, 2010.

9. Longo L, Platini F, Scardino A, Alabiso O, Vasapollo G and Tessitore L: Autophagy inhibition enhances anthocyanininduced apoptosis in hepatocellular carcinoma. Mol Cancer Ther 7: 2476-2485, 2008.

10. Chang C, Zhu YQ, Mei JJ, Liu SQ and Luo J: Involvement of mitochondrial pathway in NCTD-induced cytotoxicity in human hepG2 cells. J Exp Clin Cancer Res 29: 145, 2010.

11. Settembre C, Di Malta C, Polito VA, Garcia Arencibia M, Vetrini F, Erdin S, Erdin SU, Huynh T, Medina D, Colella P, et al: TFEB links autophagy to lysosomal biogenesis. Science 332: 1429-1433, 2011.

12. Carchman EH, Rao J, Loughran PA, Rosengart MR and Zuckerbraun BS: Heme oxygenase-1-mediated autophagy protects against hepatocyte cell death and hepatic injury from infection/sepsis in mice. Hepatology 53: 2053-2062, 2011.

13. Lewis JS, Meeke K, Osipo C, Ross EA, Kidawi N, Li T, Bell E, Chandel NS and Jordan VC: Intrinsic mechanism of estradiolinduced apoptosis in breast cancer cells resistant to estrogen deprivation. J Natl Cancer Inst 97: 1746-1759, 2005.

14. Jhunjhunwala S, Jiang Z, Stawiski EW, Gnad F, Liu J, Mayba O, Du P, Diao J, Johnson S, Wong KF, et al: Diverse modes of genomic alteration in hepatocellular carcinoma. Genome Biol 15: 436, 2014.

15. Yeh CB, Su CJ, Hwang JM and Chou MC: Therapeutic effects of cantharidin analogues without bridging ether oxygen on human hepatocellular carcinoma cells. Eur J Med Chem 45: 3981-3985, 2010.

16. Williams LA, Möller W, Merisor E, Kraus W and Rösner H: In vitro anti-proliferation/cytotoxic activity of cantharidin (Spanish Fly) and related derivatives. West Indian Med J 52: 10-13, 2003.

17. Xu Y, An Y, Wang Y, Zhang C, Zhang H, Huang C, Jiang H, Wang $\mathrm{X}$ and $\mathrm{Li} \mathrm{X}$ : miR-101 inhibits autophagy and enhances cisplatin-induced apoptosis in hepatocellular carcinoma cells. Oncol Rep 29: 2019-2024, 2013.

18. Mizushima N, Levine B, Cuervo AM and Klionsky DJ: Autophagy fights disease through cellular self-digestion. Nature 451: 1069-1075, 2008.

19. Xie JM, Li B, Yu HP, Gao QG, Li W, Wu HR and Qin ZH: TIGAR has a dual role in cancer cell survival through regulating apoptosis and autophagy. Cancer Res 74: 5127-5138, 2014.

20. Xie SQ, Zhang YH, Li Q, Xu FH, Miao JW, Zhao J and Wang CJ: 3-Nitro-naphthalimide and nitrogen mustard conjugate NNM-25 induces hepatocellular carcinoma apoptosis via PARP-1/p53 pathway. Apoptosis 17: 725-734, 2012.

21. Qi F, Inagaki Y, Gao B, Cui X, Xu H, Kokudo N, Li A and Tang W: Bufalin and cinobufagin induce apoptosis of human hepatocellular carcinoma cells via Fas- and mitochondriamediated pathways. Cancer Sci 102: 951-958, 2011.

22. Shen B, He PJ and Shao CL: Norcantharidin induced DU145 cell apoptosis through ROS-mediated mitochondrial dysfunction and energy depletion. PLoS One 8: e84610, 2013.

23. Kim I and Lemasters JJ: Mitophagy selectively degrades individual damaged mitochondria after photoirradiation. Antioxid Redox Signal 14: 1919-1928, 2011.

24. Zhang R, Humphreys I, Sahu RP, Shi Y and Srivastava SK: In vitro and in vivo induction of apoptosis by capsaicin in pancreatic cancer cells is mediated through ROS generation and mitochondrial death pathway. Apoptosis 13: 1465-1478, 2008

25. Kang JY, Eggert M, Mouli S, Aljuffali I, Fu X, Nie B, Sheil A, Waddey K, Oldham CD, May SW, et al: Pharmacokinetics, antitumor and cardioprotective effects of liposome-encapsulated phenylaminoethyl selenide in human prostate cancer rodent models. Pharm Res 32: 852-862, 2014.

26. Ott M, Gogvadze V, Orrenius S and Zhivotovsky B: Mitochondria, oxidative stress and cell death. Apoptosis 12: 913-922, 2007. 